\title{
Minimum node degree of $k$-connected vehicular ad hoc networks in highway scenarios
}

\author{
Wei Xiong ${ }^{1,2^{*}}$ (D), Jingjing $\mathrm{Xu}^{1,2}$, Yan $\mathrm{Li}^{2,3}$, Nan Zhao ${ }^{1,2}$, Xiangkui Wan ${ }^{1,2}$ and Jin Liang ${ }^{4}$
}

\begin{abstract}
A vehicular ad hoc network (VANET) is a specific type of mobile ad hoc networks (MANETs); it can provide direct or multi-hop vehicle-to-vehicle (V2V), vehicle-to-roadside (V2R), vehicle-to-pedestrian (V2P), and vehicle-to-internet (V2I) communications based on the pre-existing road layouts. The emerging and promising VANET technologies have drawn tremendous attention from the government, academics, and industry over the past few years and have been increasingly available for a large number of cutting edge applications that can be classified into road safety, traffic efficiency, and infotainment categories. Due to the unique characteristics of VANETs, such as high mobility with an organized but constrained pattern, and diverse radio propagation conditions, the conventional researches dedicated for general MANETs cannot be directly applied to VANETs. This paper presents an analytical framework to investigate the minimum node degree of $k$-connected VANETs, with a homogeneous range assignment in highway scenarios. We simulate the mobility patterns with realistic vehicular traces, model the network topology as a two-path fading geometric random graph, and conduct extensive experiments on the derived analytical results. Through a combination of mathematical modeling and simulations, we derive a probabilistic bound for the minimum node degree of a homogeneous vehicular ad hoc network in highway scenarios. The analytical framework is useful in the study of connectivity and estimation of performance in one-dimensional vehicular ad hoc networks.
\end{abstract}

Keywords: Vehicular ad hoc networks (VANETs), Minimum node degree, Network connectivity

\section{Introduction}

The internet of vehicles (IoV) is a complex integrated network system that interconnects people within and around vehicles, intelligent systems on board vehicles, and various cyber-physical systems in urban environments $[1,2]$.

A vehicular ad hoc network (VANET) is emerging as an important part of the IoV and goes beyond telematics or intelligent transportation systems (ITS) by integrating vehicles, sensors, and mobile devices into a global network.

The promising VANET technologies have enabled a large number of road safety [3-5], traffic efficiency [6, 7],

\footnotetext{
*Correspondence: xw@mail.hbut.edu.cn

1 Hubei Collaborative Innovation Center for High-efficient Utilization of Solar Energy, Hubei University of Technology, Wuhan 430068, People区 Republic of China

${ }^{2}$ School of Electrical and Electronic Engineering, Hubei University of

Technology, Wuhan 430068, People区 Republic of China

Full list of author information is available at the end of the article
}

and infotainment [8-11] applications via direct or multihop vehicle-to-vehicle (V2V), vehicle-to-roadside (V2R), vehicle-to-pedestrian (V2P), and vehicle-to-internet (V2I) communications.

Recently, good progress has been made in the development of dedicated medium access control (MAC) [12-14], routing [15-18], and security $[19,20]$ protocols for vehicular ad hoc networks, but less work has been done to investigate their fundamental properties and network topologies.

VANETs have several characteristics that distinguish them from mobile ad hoc networks (MANETs), e.g., high mobility with an organized but constrained pattern, and diverse radio propagation conditions [21]; the corresponding network topology changes rapidly, which in turn leads to frequent network fragmentation, and end-to-end path is not guaranteed. Moreover, the quasilinear topology of VANETs may reduce the possible link redundancy [22].

\section{包}


In this paper, we investigate an essential prerequisite, namely the minimum node degree, of $k$-connected vehicular networks, with a homogeneous range assignment in highway scenarios. The main contributions of this paper are twofold:

- We propose a notion of arc-edge rectangular effective area contributed for networking and reduce the computational complexity.

- We derive a closed formula for the probability that each vehicle in the network has at least $k$ neighbors $(k=1,2,3, \cdots)$.

Our analyses are of practical value for researchers and developers who perform design or implementation of real-life vehicular ad hoc systems.

The remainder of this paper is organized as follows: Section 2 describes the VANET system model. Section 3 derives the probability that each vehicle in the network has at least $k$ neighbors $(k=1,2,3, \cdots)$. We conduct extensive experiments and thereby verify our analytical expressions in Section 4. Section 5 outlines the related work and compares them to our contributions. Finally, Section 6 concludes the paper and gives ideas for further research.

\section{VANET system model}

\subsection{Vehicular mobility model}

Vehicular mobility has strong impact on the performance of protocols proposed at various layers and the feasibility of applications over VANETs. Realistic representation of vehicular mobility requires accurate microscopic mobility modeling, real-world road topology, and real database traffic demand modeling [23].

Studies have shown that widely used mobility models for MANETs, such as random waypoint or random direction, are not suitable to simulate vehicular movement, which is constrained to a large extent by highway patterns, traffic situations, and driver behaviors. The street-bounded mobility of each individual vehicle as well as the interactions with respect to other vehicles must be taken into considerations, and such behaviors as car-following, overtaking, and lane-changing need to be modeled in detail.

The simulation of urban mobility (SUMO), ${ }^{1}$ which is a free and open-source space-continuous discrete-time microscopic traffic simulator, is used to generate highway mobility traces. The movement of each vehicle is determined based on the source/destination matrix provided as an input to the simulator. The behavior of each driver is implemented based on the surrounding vehicles via Krauss' car-following model, which regulates its acceleration, and Krajzewicz's lane-changing model, which regulates its overtaking decisions [23].
The parameters that determine a driver's acceleration and overtaking decisions include the distance to the leading vehicle, the traveling speed, the acceleration and deceleration profiles, and the size of vehicles. We record the instantaneous position, speed, acceleration, and lane information of each individual vehicle at each time instant, corresponding to vehicular traffic flows during $60 \mathrm{~s}$ in a straight bidirectional quad-lane highway scenario with different settings of traffic density, from 2 to 15 vehicles per kilometer in each lane. The two traveling directions are separated by a median zone. Each direction is then further divided into a normal-speed lane and an overtaking lane. Without loss of generality, we illustrate our highway scenario model in Fig. 1.

The lane changes will take place between the two adjacent intervals, $0.5 \mathrm{~s}$, since the raw data only provides the two-tuples (position, lane) to describe the vehicular locations on highways. The second element of each vehicle is for most purposes quantized with the midline of each lane, which is mapped according to an assumed but realistic values for highways in mainland China. Referring to field measurements, we stipulate that the lane width and median width are 3.75 and $2.00 \mathrm{~m}$, respectively. As depicted in Fig. 1, the quantization formula is as follows:

$$
Y= \begin{cases}0.5 \times \text { LaneWidth, } & \text { Lane }=1 \\ 1.5 \times \text { LaneWidth, } & \text { Lane }=2 \\ 2.5 \times \text { LaneWidth }+ \text { MedianWidth }, & \text { Lane }=3 \\ 3.5 \times \text { LaneWidth }+ \text { MedianWidth, } & \text { Lane }=4 .\end{cases}
$$

\subsection{Radio propagation model}

When a signal passes through the wireless channel, it will suffer from the effects of a variety of fading. Radio channel modeling, which is used to predict the received signal power, for various propagation environments with a sufficient grade of accuracy is therefore a demanding task. Commonly, the modeling is divided into large-scale and small-scale radio propagation models [21]. The large-scale fading, due to path loss of signals as a function of distance and shadowing by large obstacles such as buildings and hills, occurs as the mobile moves through a distance of the order of several hundreds or thousands of meters and is typically frequency independent. The small-scale fading, due to the constructive and destructive interference of the multiple signal paths between the transmitter and receiver, occurs at either spatially (the order of the carrier wavelength) or temporally (the order of a few seconds) and is frequency dependent.

While there are many sophisticated statistical models to describe multi-path fading in radio propagation (such as Rayleigh, Ricean, and Nakagami distributions), several studies have also shown that the two-ray ground reflection model, which considers both a direct propagation path and a ground reflection path, gives a good result 


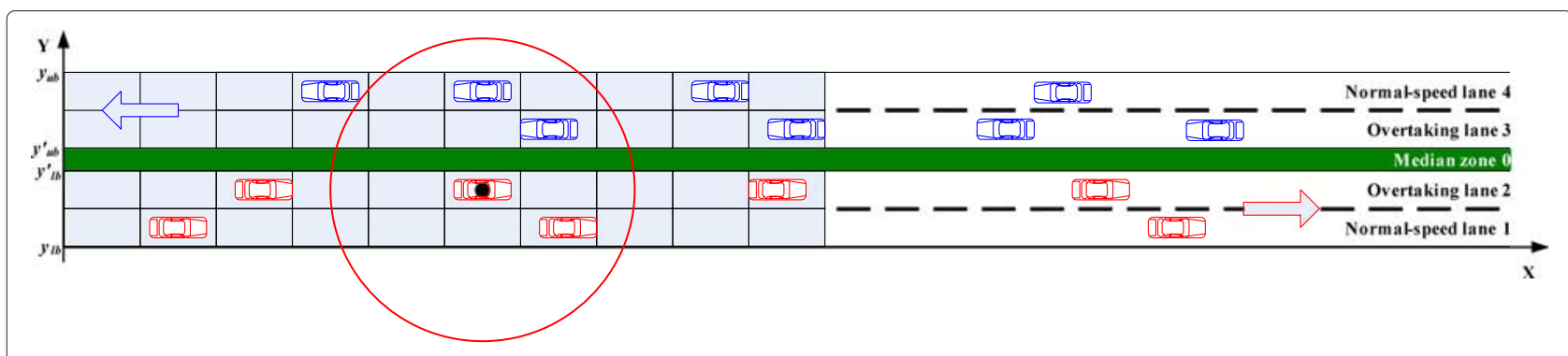

Fig. 1 Highway scenario model

for dedicated short-range inter-vehicle communications at $5.9 \mathrm{GHz}$ band [24-26]. We adopt the following generalized log-distance path loss model to reproduce wireless links between the transmitter and receiver:

$$
\left[P_{\mathrm{rx}}(d)\right]_{\mathrm{dB}}=\left[P_{\mathrm{tx}}\right]_{\mathrm{dB}}-\left[\mathrm{PL}\left(d_{0}\right)\right]_{\mathrm{dB}}-10 \beta \log _{10}\left(\frac{d}{d_{0}}\right),
$$

where $P_{\mathrm{tx}}$ and $P_{\mathrm{rx}}(d)$ are the transmitted and received signal power at distance $d$ in between, $d_{0}$ is the close-in reference distance, and $\beta$ is called the path loss exponent, which depends on the environment. In vehicular communication scenarios, the path loss exponents ranging from 1.4 to 3.5 and from 2.8 to 5.9 have been reported in lineof-sight (LOS) and non-line-of-sight (NLOS) situations, respectively $[24,25]$. The wireless transmission range $r_{0}$ of each node can then be calculated as the equivalent transmission power using a receiving threshold, $P_{\mathrm{rx}, \mathrm{th}}$, also referred to as the reception sensitivity. When the received signal power is below the receiving threshold, we believe that there is no wireless link between the two nodes. Only omnidirectional antennas and bidirectional radio links are considered in this paper.

In summary, such a radio link model is not very sophisticated, but it is well-suited for our investigations on the fundamental properties and network topologies (e.g., degree distribution, network connectivity). Furthermore, the computational complexity does not allow us to simulate detailed statistical fading effects in a large-scale network simulation.

\section{Minimum node degree}

In order to better present the following sections, we briefly introduce some definitions of graph theory and define the nomenclature used in this paper. A graph $G$ is said to be connected, and if for each pair of nodes, there exists a path between them; otherwise, it is disconnected. The degree of a node $u$, denoted as $d_{G}(u)$, is the number of neighbors of node $u$, i.e., the number of its links. A node of degree $d_{G}(u)=0$ is isolated, i.e., it has no neighbors. The minimum node degree of a graph $G$ is denoted as $\delta(G)=\min _{\forall u \in G}\left\{d_{G}(u)\right\}$.
Empirical observations in traffic science show that under free traffic flow conditions, the time headway $h_{t}$ follows an exponential distribution [27]. Supposing that the traveling speed $v_{s}$ of vehicles is uniformly distributed, the space headway $h_{s}$ and the time headway $h_{t}$ follow the same distribution. In a previous work for VANETs, the space headway is usually modeled as exponential distribution [28]. Thus, the probability that a node has no neighbors within its communication range $r$, viz., it is isolated, is $\operatorname{Pr}\{\kappa=0\}=e^{-\lambda A}$. The probability that a node has at least $k$ neighbors within its communication range is

$$
\begin{aligned}
\operatorname{Pr}\{\kappa \geq k\} & =1-\sum_{j=0}^{k-1} \operatorname{Pr}\{\kappa=j\} \\
& =1-e^{-\lambda A} \sum_{j=0}^{k-1} \frac{(\lambda A)^{j}}{j !}
\end{aligned}
$$

where $\lambda=1 /\left(v_{s} \cdot h_{t} \cdot m\right.$. LaneWidth $)$ and $m$ is the number of lanes. Due to the specific characteristics of VANETs, the effective area $A$ contributed for networking is the intersection area of highway lanes and radio coverage. Considering the space limitation, we simply analyze such a case that the radio communication range is long enough, viz., $r>3.5 \times$ LaneWidth + MedianWidth.

Let $A_{1}$ and $A_{2}$ be the intersection areas of highway lanes and radio coverage centered at the middle of the normal-speed lane $(i=1)$ and overtaking lane $(i=2)$, respectively. We then have

$$
\begin{aligned}
A_{i}= & \int_{0}^{4 \text { LaneWidth+MedianWidth }} \int_{x_{i}-\sqrt{r^{2}-\left(y-y_{i}\right)^{2}}}^{x_{i}+\sqrt{r^{2}-\left(y-y_{i}\right)^{2}}} d x d y \\
& -\int_{\text {2LaneWidth }}^{\text {2LaneWidth+MedianWidth }} \int_{x_{i}-\sqrt{r^{2}-\left(y-y_{i}\right)^{2}}}^{x_{i}+\sqrt{r^{2}-\left(y-y_{i}\right)^{2}}} d x d y \\
= & c_{i} r^{2},(i=1,2)
\end{aligned}
$$


where

$$
\begin{aligned}
c_{i}= & {\left[\arcsin \left(\frac{y_{u b}-y_{i}}{r}\right)-\arcsin \left(\frac{y_{l b}-y_{i}}{r}\right)\right.} \\
& +\frac{y_{u b}-y_{i}}{r} \sqrt{1-\left(\frac{y_{u b}-y_{i}}{r}\right)^{2}} \\
& \left.-\frac{y_{l b}-y_{i}}{r} \sqrt{1-\left(\frac{y_{l b}-y_{i}}{r}\right)^{2}}\right]_{\left(r, 2 \mathrm{LW}, 2 \mathrm{LW}+\mathrm{MW}, y_{i}\right)}^{\left(r, 0,4 \mathrm{LW}+\mathrm{MW}, y_{i}\right)}, i=1,2
\end{aligned}
$$

We can clearly see from Fig. 2 that the differences between the normal-speed lane coefficient $c_{1}$ and overtaking lane coefficient $c_{2}$ vanish while the communication range $r$ increases. Let $c=\left(c_{1}+c_{2}\right) / 2$, hence the probability that a node has at least $k$ neighbors within its communication range $r$ is

$$
\operatorname{Pr}\{\kappa \geq k\}=1-e^{-\lambda c r^{2}} \sum_{j=0}^{k-1} \frac{\left(\lambda c r^{2}\right)^{j}}{j !}
$$

We also notice that the log-log graph of the average lane coefficient $c$ and communication range $r$ yields a very good straight line, suggesting that they have a power law relationship, i.e., $c \sim \Theta\left(r^{-\gamma}\right)$, where the power-law data fitting $\gamma=0.9988$ and the corresponding residual error $\varepsilon=1.0575 \times 10^{-6}$.

The objective is to achieve a network in which none of the $n$ nodes is isolated, i.e., $\delta(G)>0$. Assuming the statistical independence, the probability of no isolated nodes within the entire network is

$$
\operatorname{Pr}\{\delta(G)>0\}=\left(1-e^{-\lambda c r^{2}}\right)^{n}
$$

and more generally, the probability that each node has at least $k$ neighbors within the entire network is

$$
\operatorname{Pr}\{\delta(G) \geq k\}=\left[1-e^{-\lambda c r^{2}} \sum_{j=0}^{k-1} \frac{\left(\lambda c r^{2}\right)^{j}}{j !}\right]^{n}
$$

\section{Experimentation and analysis}

In this section, we verify the derived analytical results with the realistic vehicular network model. We make extensive experiments on the five selected highway scenarios, each has a different density of traffic flows with two lanes per direction. The numerical characteristics of vehicles in the experimental scenarios are listed in Table 1.

Given that the length of the highway is $12 \mathrm{~km}$, the $3 \mathrm{~d}$ mesh grids of the theoretical probabilities that each node in the network has at least 1,2 , or 3 neighbors are illustrated in Figs. 3a, 4a, and 5a, respectively. The probability that each node in the network has at least $k$ neighbors, $\operatorname{Pr}\{\delta(G) \geq k\}$, increases with respect to the number of vehicles or radio communication ranges, while decreases with respect to the minimum node degree, $k$, for a given $(n, r)$ scenarios.

We regard the mobility scenarios as a series of snapshots, in which each vehicle is denoted as a vertex. The homogeneous transmission range of each node starts from $1 \mathrm{~m}$ and then increases by unit step until each vehicle in the network has at least 1,2 , or 3 neighbors. We define the probability that each node has at least $k$ neighbors as:

$$
\operatorname{Pr}\{\delta(G) \geq k\}=\frac{\text { Number of nodes with at least } k \text { neighbors }}{\text { Number of nodes within the entire network }}
$$

If none of the nodes in the network has at least $k$ neighbors, then the probability $\operatorname{Pr}\{\delta(G) \geq k\}=0$. If all nodes

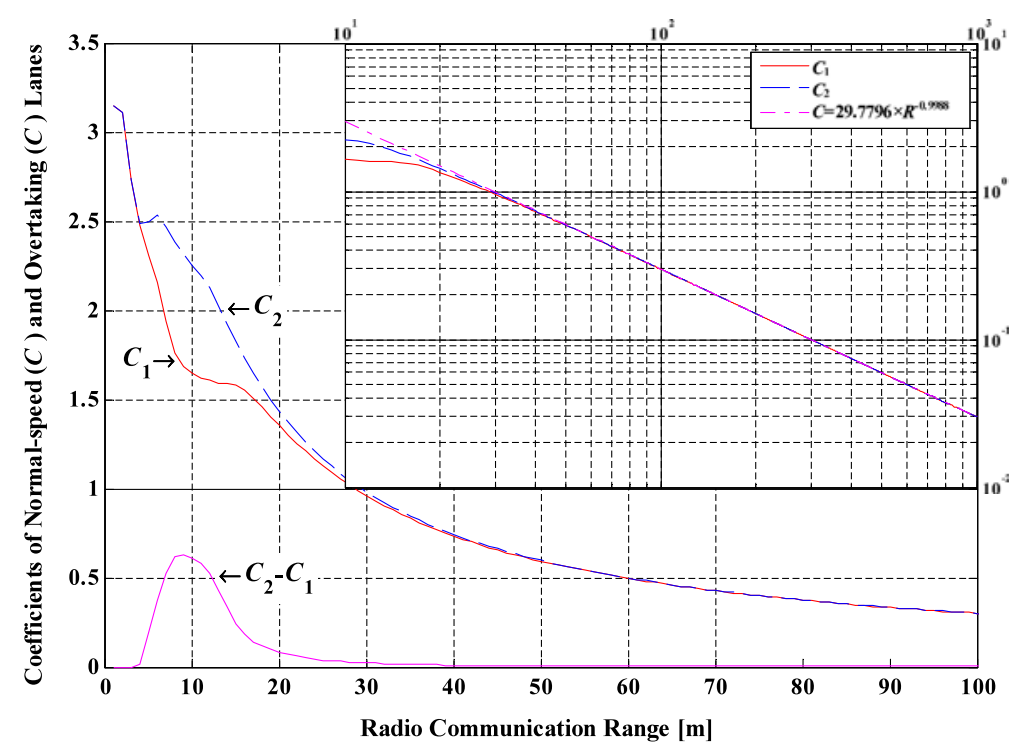

Fig. 2 Coefficients of normal-speed and overtaking lanes 
Table 1 Numerical characteristics of experimental scenarios

\begin{tabular}{llllll}
\hline Scenario & $\begin{array}{l}\text { Traffic density } \\
(\text { veh./(km.lane) })^{\mathrm{a}}\end{array}$ & Duration $(\mathrm{s})$ & Number of vehicles ${ }^{\mathrm{b}}$ & Speed $(\mathrm{km} / \mathrm{h})^{\mathrm{b}}$ & Scenario length $(\mathrm{m})$ \\
\hline Scene 1 & $2-2$ & 60 & $82 / 92 / 102$ & $75 / 140 / 243$ & 11,600 \\
Scene 2 & $2-6$ & 60 & $189 / 203 / 212$ & $66 / 132 / 240$ & 11,881 \\
Scene 3 & $6-6$ & 60 & $276 / 305 / 326$ & $66 / 128 / 223$ & 12,113 \\
Scene 4 & $6-11$ & 60 & $379 / 420 / 441$ & $49 / 122 / 223$ & 12,336 \\
Scene 5 & $15-15$ & 60 & $725 / 749 / 787$ & $46 / 111 / 207$ & 12,562 \\
\hline
\end{tabular}

aThe two numbers denote vehicle distribution density to the left-right direction

${ }^{b}$ The three numbers denote the minimum/mean/maximum values of that parameter

have at least $k$ neighbors, then the probability $\operatorname{Pr}\{\delta(G) \geq$ $k\}=1$.

The experimental probabilities that each node in the network has at least 1,2 , or 3 neighbors, as well as the corresponding theoretical probabilities calculated from Eq. (5) are illustrated in Figs. 3b, 4b, and 5b, respectively. We can clearly see that the numerical results are in good agreement with those obtained from experiments.

\section{Related work}

A large number of studies concerning network connectivity modeling and analysis of one- or two-dimensional MANET have been reported in the recent two decades. All the research work aims at providing a means to estimate the grade of service (GoS) measured in network connectivity probability. One of the first papers on critical connectivity phenomena in a multi-hop radio network, modeled by a spatial Poisson point process, was studied by Cheng and Robertazzi [29] in 1989. It investigated the relations between transmission range $r$, node density $\rho$, and network connectivity in the context of "broadcast percolation" mechanism. Philips et al. [30] studied the connectivity properties of a homogeneous Poisson distributed model and confirmed that $\rho \pi r^{2}$, the expected number $k$ of nearest neighbors of a node, should be subjected to the logarithm of the bounded area $A$ rather than the wellknown "magic number" of 6 . They tightened the critical value to $2.195<k<10.526$. Piret [31] demurred to some of the results derived in [30], and proved the critical range of one-dimensional network connectivity may be different from that of one-dimensional network coverage, and conjectured that the two critical ranges in two-dimensional situations are also different. Gupta and Kumar [32] performed a fundamental work on the asymptotic connectivity of wireless networks, in which nodes are uniformly and independently distributed in a unit disc area. They derived the critical power that a node in a network needs to transmit in order to guarantee that the network is connected with high probability (w.h.p.) as the number of nodes goes to infinity. Experimental results demonstrated that if

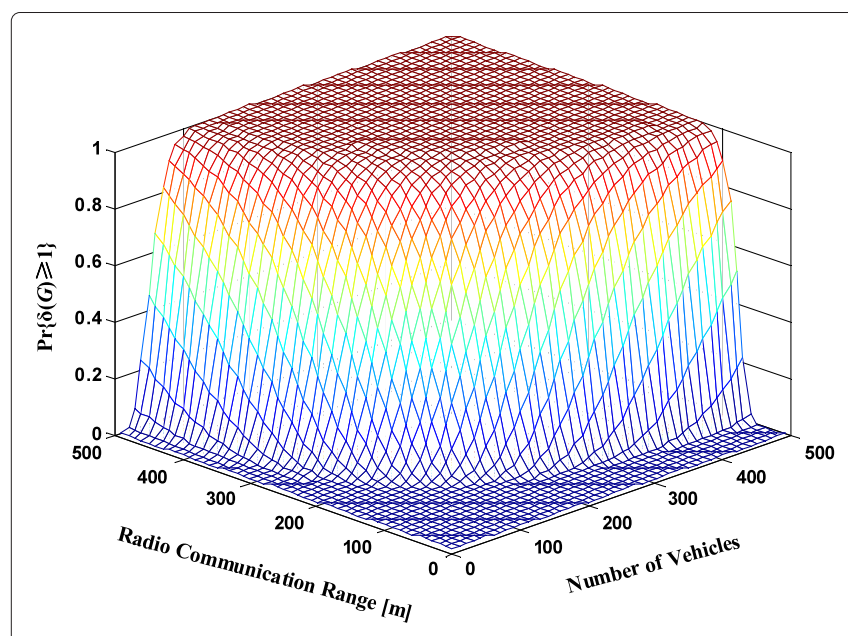

(a) Theoretical probability $\operatorname{Pr}\{\delta(G) \geq 1\}$

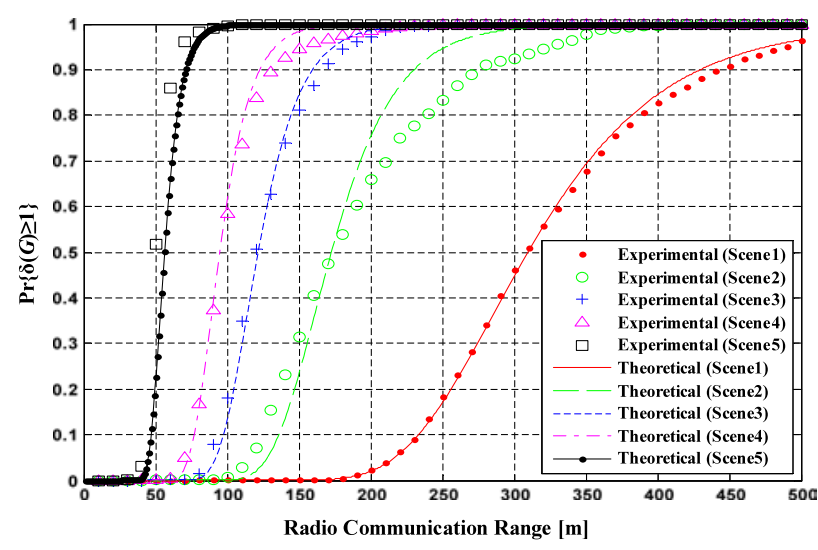

(b) Experimental vs. Theoretical probabilities

Fig. 3 Probability that each node has at least 1 neighbor within the entire network. a Theoretical probability $\operatorname{Pr}\{\delta(G) \geq 1\}$ and $\mathbf{b}$ experimental vs. theoretical probabilities 


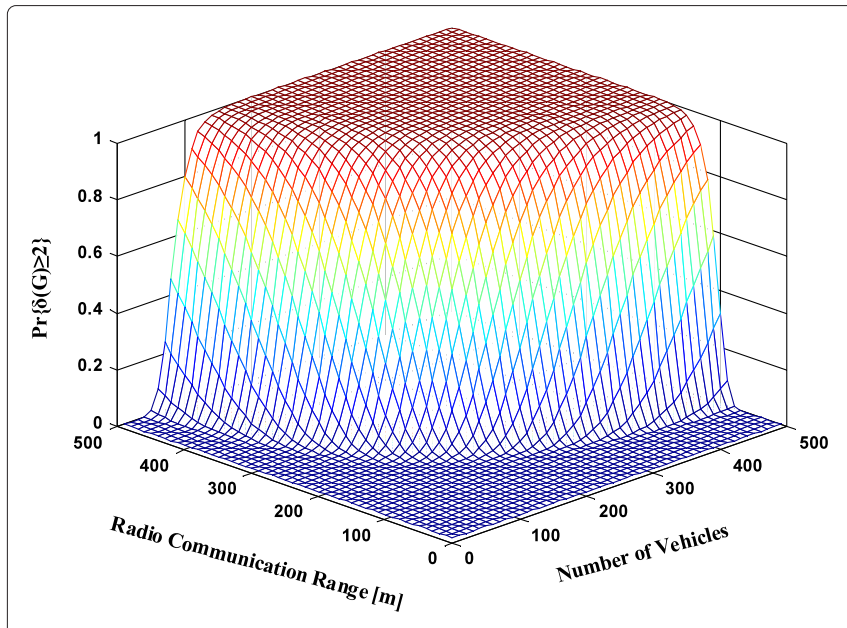

(a) Theoretical probability $\operatorname{Pr}\{\delta(G) \geq 2\}$

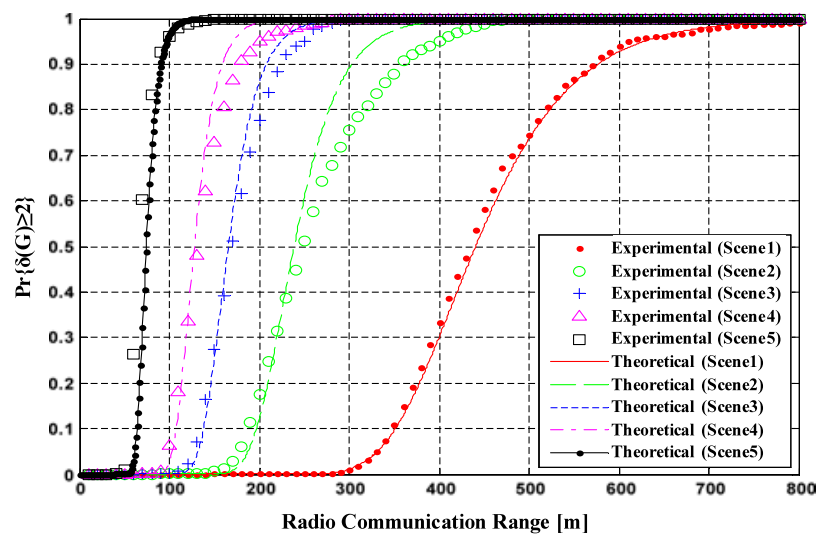

(b) Experimental vs. Theoretical probabilities

Fig. 4 Probability that each node has at least 2 neighbors within the entire network. a Theoretical probability $\operatorname{Pr}\{\delta(G) \geq 2\}$ and $\mathbf{b}$ experimental vs. theoretical probabilities

$\pi r^{2}(n)=(\log (n)+c(n)) / n$, then $P_{\text {conn }}(n, r(n)) \rightarrow 1$, if and only if (iff) $c(n) \rightarrow+\infty$. Sánchez et al. [33] presented a minimal spanning tree (MST) algorithm to calculate the critical transmission range (CTR) that allows maintaining full network connectivity. The simulations indicated that there was no dependence on the tested mobility models. Santi et al. [34] considered range assignment problems under a homogeneous probabilistic model and established the lower and upper bounds of the network connectivity probability. The derived necessary and sufficient conditions to ensure a strongly connected network, in which nodes are uniformly and independently distributed in a one-dimensional case, are $n r / l$ and $n r$, respectively; and those for two- and three-dimensional cases are investigated via simulations. Krishnamachari et al. [35] discussed by means of simulations that a certain of the monotone network properties, such as connectivity, exhibited the critical threshold effect, above which the desired global network property existed w.h.p., while below which the desired global network property might exist with low probability. Such behavior is akin to "phase transition" in Physics and "zero-one" law in Bernoulli random graphs. Bettstetter [36] investigated the two fundamental characteristics of a wireless multi-hop network: its minimal

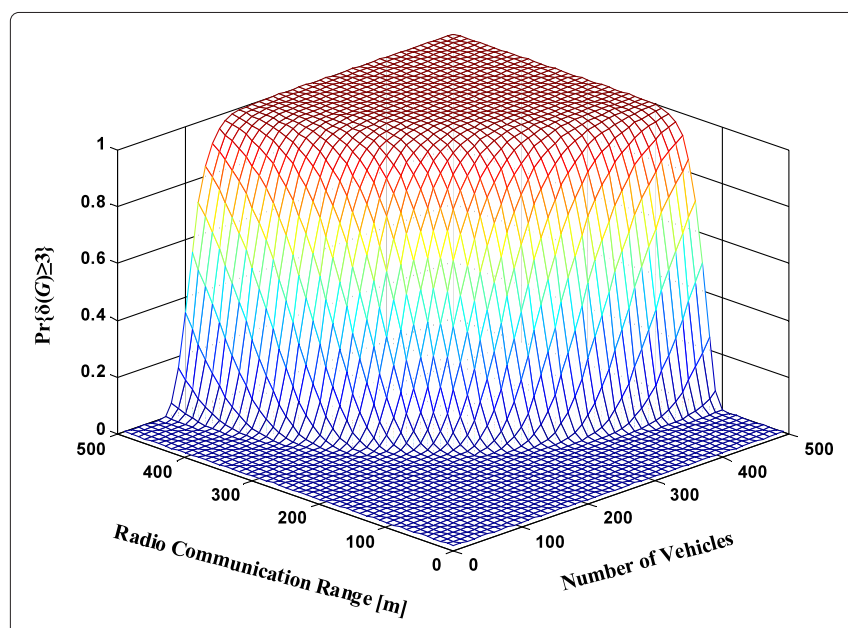

(a) Theoretical probability $\operatorname{Pr}\{\delta(G) \geq 3\}$

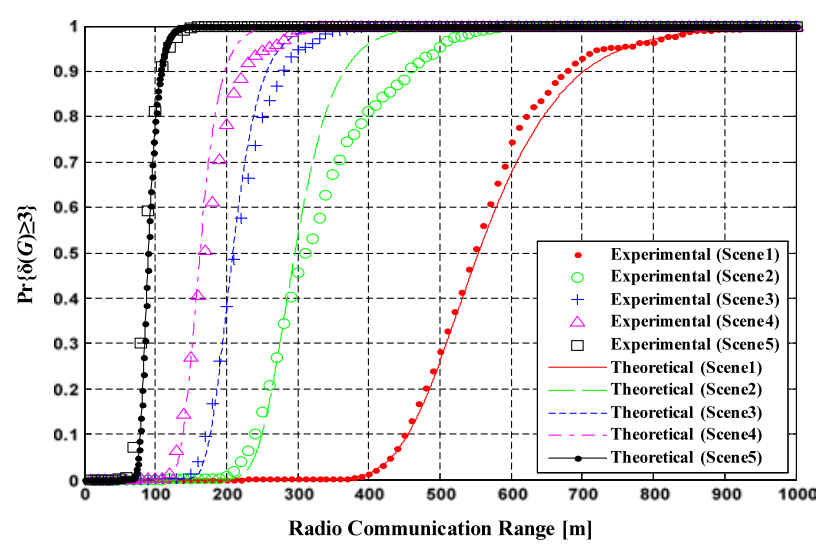

(b) Experimental vs. Theoretical probabilities

Fig. 5 Probability that each node has at least 3 neighbors within the entire network. a Theoretical probability $\operatorname{Pr}\{\delta(G) \geq 3\}$ and $\mathbf{b}$ experimental vs. theoretical probabilities 
node degree and its $k$-connectivity. The authors derived an analytical expression to determine the required $(n, r)$ pairs that achieved an almost surely (a.s.) $k$-connected network with homogeneous Poisson distributed nodes in two dimensions. Bettstetter [37] deepened the research work on the $k$-connected networks with homogeneous and inhomogeneous range assignments. To examine the impact of nodal mobility on $k$-connectivity issues, refs. $[38,39]$ considered the border effects. Xue and Kumar [40] resolved the number of neighbors needed for wireless ad hoc network connectivity and indicated that in a network with $n$ randomly distributed nodes, each node should be connected to $\Theta(\log (n))$ nearest neighbors. When each node connects to more than $5.1774 \log (n)$ nearest neighbors, the network is asymptotically connected w.h.p. as $n$ increases. However, if each node has less than $0.074 \log (n)$ nearest neighbors, then the entire network is asymptotically disconnected w.h.p. as $n$ increases.

Most literature on the connectivity of mobile ad hoc networks only deal with one- or two-dimensional asymptotic results in the number of nodes in the network. Desai and Manjunath [41] first analyzed the connectivity in a finite ad hoc network with nodes uniformly distributed in one-dimension and derived a closed-form equation to compute the network connectivity probability. Wang et al. [42] also derived a generic formula for the probability that a one-dimensional MANET is connected. They investigated some topological characteristics of connected ad hoc networks, especially the derivations of necessary condition for network connectivity in oneand two-dimensions. Dousse et al. [43] considered the connectivity property in pure ad hoc and hybrid networks with sparse density. They found that the introduction of fix infrastructure (e.g., base stations) into the network can dramatically improve the network connectivity in onedimensional and strip cases with finite width and infinite length, but that does not work for two-dimensional cases. Santi and Blough [44] studied the relative magnitude of transmission range $r$, the number of nodes $n$, and size scale $l$ of the network to create a connected graph with high probability, and the primary analytical result indicated that a one-dimensional network with nodes uniformly distributed would be connected, iff $n r \sim \Theta(l \cdot \log (l))$, which complements the lower and upper bounds on the range assignment that were published in [34]. For the most commonly used mobility model in the simulation of ad hoc networks, Bettstetter et al. [45] presented an in-depth analysis on the node distribution of the random waypoint (RWP) mobility model. They concluded that the resulting distribution consists of weighted sum of the three independent components - the static, the pause, and the mobility-and also derived an exact formula of the asymptotically stationary distribution for movement on a line segment and an accurate approximation for a square area. Panchapakesan and Manjunath [46] investigated the CTR for connectivity in dense ad hoc radio networks, while Santi and Blough [47] analyzed the CTR for connectivity in a sparse wireless ad hoc network. Foh and Lee [48] and Foh et al. [49] emphasized the connectivity for one-dimensional MANET; they obtained the numerical expression, $P_{n}(l)=\left(1-(1-1 / l)^{n}\right)^{(n+1)}, n \geq l-1$, to generate a fully connected network given a certain number of nodes randomly and uniformly distributed in a line.

Viewing the literature both here and abroad, researchers have mainly focused on the connectivity aspect of oneand two-dimensional MANETs in which nodes are distributed uniformly or according to the Poisson point process. For the nodal mobility, the RWP and random direction models were primarily adopted in the simulations; but those models could not commendably reflect the actual mobility characteristics of the vehicles. So far, just a few papers have investigated the connectivity problems in VANETs. Chen et al. [50] validated that store-andforward mechanism could conduce to successful message delivery among vehicles at a high speed. Using vehicle mobility traces generated from a traffic micro-simulator, corridor simulator (CORSIM), the authors found that by measuring average delivery time that the mobility of vehicles on bidirectional highways with multi-lanes, each can significantly decrease the end-to-end delay. Füßler et al. [51] investigated the usage of ad hoc routing algorithms (e.g., DSR and GPSR) for data exchanging among vehicles. By dint of realistic vehicular mobility patterns of highway traffic scenarios generated from a well validated DaimlerChrysler-internal driver behavior traffic simulation tool, FARSI, the experimental results indicated that network partitioning would frequently occur even if the density of vehicles were much higher, but such a phenomenon could be restrained by increasing nodes communication range as well as by internetworking with countermove vehicles. Rudack et al. [52] then analyzed the impact of vehicular traffic dynamics on MAC protocols for ad hoc networks. Based on analytical investigations of topology dynamics and on realistic traffic simulations, the authors investigated possible probability distribution of link durations in various highway scenarios. Artimy et al. [53] explored the effects of a multi-lane, unidirectional free-flow traffic on the connectivity of IVC networks and concluded that vehicle density, relative velocity, and number of lanes were some of the key factors. The effect of the spatial distance between nodes, however, depended on the radio transmission range. Furthermore, they also observed that the probability distribution of link durations resembled a power law function. Artimy et al. [54] discussed the range assignment problems in VANET and determined via simulations that the minimal transmission range (MTR) was required to maintain the 
connectivity of various closed-loop shape vehicular networks. The results indicated that the value of the MTR would decrease and stay around a certain fixed value as the density of vehicles increased up to the critical density. When traffic jams occurred, the value of the MTR would remain the same for all the possible densities. That paper finally suggested that the communication range should be set to $r \geq 0.12 l$ (about $450 \mathrm{~m}$ ), at which the number of network partitions in all the five investigated road scenarios was less than 5. Artimy et al. [55] examined the impact of the non-homogeneous distribution of vehicles (e.g., in traffic jams) on the connectivity of VANET and provided a tighter estimation for the lower bound of the MTR in a finite-length highway scenario. Besides, the authors presented an empirical estimation for the upper bound of the MTR via simulations. Ukkusuri and Du [28] developed analytical expressions for VANET connectivity with respect to the number of reachable neighbors on a unidirectional highway segment. The analytical results revealed that increasing with the individual reachable neighbors would improve the connectivity of the entire network, and at the same time, the boundary effect would be impaired. The network was asymptotically connected if $k \sim \Theta\left(\log \left(n^{\xi}\right)\right), \xi>2$.

\section{Conclusions}

The physical topology connectivity poses a great challenge to the emerging and promising VANETs, especially in highway scenarios. The existence of isolated nodes not only restricts the information disseminations within the entire network but also exacerbates the end-to-end delay. This paper investigates an essential prerequisite, namely the minimum node degree, of $k$-connected vehicular ad hoc networks, with a homogeneous range assignment in highway scenarios. Among other things, we simulated the mobility of vehicles with realistic trace data, modeled the network as a geometric random graph, and also derived an analytical algorithm to estimate the probability that each node in the network has at least $k$ neighbors. Extensive experiments were undertaken to verify the derived algorithm, with border effect, via realistic mobility traces.

The main contributions of this paper are considered as follows:

1. We proposed the notion of arc-edge rectangular effective area contributed for networking, namely, the intersection area of highway lanes and radio coverage, and tightened the effective area $A$ from $\pi r^{2}$ to $c r^{2}$, where $c<\pi$.

2. We derived a closed formula to estimate the probability that each vehicle has at least $k$ neighbors, i.e., the network has a minimum node degree $\delta(G) \geq k$, is given by $\operatorname{Pr}\{\delta(G) \geq k\}=\left[1-e^{-\lambda c r^{2}} \sum_{j=0}^{k-1} \frac{\left(\lambda c r^{2}\right)^{j}}{j !}\right]^{n}$.
The work in this paper can be extended in several directions. One possible extension is to consider different distribution models for vehicles deployed on highways and forming the network. One, however, must bear in mind that the space-and-time distribution of vehicles in real life cannot be absolutely random. Different models are needed if the heterogeneous range assignment is taken into consideration. The $k$-connectivity and data dissemination issues are the future work for further investigation.

\section{Endnote}

${ }^{1}$ SUMO: http://sumo.sourceforge.net.

\section{Competing interests}

The authors declare that they have no competing interests.

\section{Acknowledgements}

This work was supported by the National Natural Science Foundation of China (61471162, 61501178 and 61571182), the Natural Science Foundation of Hubei Province of China (2015CFB646), the Major Science and Technology Program of Guangdong Province of China (2013A022100017), the Next Generation Internet Technology Innovation Project of CERNET (NGII20150501), the Open Foundation of Hubei Collaborative Innovation Center for High-efficient Utilization of Solar Energy (HBSKFZD2014011), and the Scientific Research Foundation of Hubei University of Technology (BSQD12022).

\section{Author details}

${ }^{1}$ Hubei Collaborative Innovation Center for High-efficient Utilization of Solar Energy, Hubei University of Technology, Wuhan 430068, People区 Republic of China. ${ }^{2}$ School of Electrical and Electronic Engineering, Hubei University of Technology, Wuhan 430068, People囚 Republic of China. ${ }^{3}$ Faculty of Health, Engineering and Sciences, University of Southern Queensland, Toowoomba 4350, Australia. ${ }^{4}$ Guangdong Biolight Meditech Co. Ltd., Zhuhai 510006 , People苗 Republic of China.

\section{Received: 20 July 2015 Accepted: 15 January 2016}

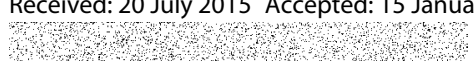

References

1. F Yang, S Wang, J Li, Z Liu, Q Sun, An overview of internet of vehicles. China Commun. 11(10), 1-15 (2014). doi:10.1109/cc.2014.6969789

2. J Wan, D Zhang, S Zhao, LT Yang, J Lloret, Context-aware vehicular cyber-physical systems with cloud support: architecture, challenges, and solutions. IEEE Commun. Mag. 52(8), 106-113 (2014). doi:10.1109/mcom. 2014.6871677

3. W Guan, J He, C Ma, Z Tang, Y Li, Adaptive message rate control of infrastructured DSRC vehicle networks for coexisting road safety and non-safety applications. Int. J. Distributed Sensor Netw. (2012). doi:10.1155/2012/134238

4. J He, Z Tang, T OWarrell, TM Chen, Performance analysis of DSRC priority mechanism for road safety applications in vehicular networks. Wireless Commun. Mobile Comput. 11(7, SI), 980-990 (2011). doi:10.1002/wcm.821

5. HT Cheng, H Shan, W Zhuang, Infotainment and road safety service support in vehicular networking: from a communication perspective. Mech. Syst. Signal Process. 25(6), 2020-2038 (2011). doi:10.1016/j.ymssp. 2010.11.009

6. J Liu, J Wan, Q Wang, D Li, Y Qiao, H Cai, A novel energy-saving one-sided synchronous two-way ranging algorithm for vehicular positioning. Mobile Netw. Appl. 20(5), 661-672 (2015). doi:10.1007/s11036-015-0604-5

7. I Leontiadis, G Marfia, D Mack, G Pau, C Mascolo, M Gerla, On the effectiveness of an opportunistic traffic management system for vehicular networks. IEEE Trans. Intell. Trans. Syst. 12(4), 1537-1548 (2011). doi:10.1109/tits.2011.2161469

8. M-K Jiau, S-C Huang, J-N Hwang, AV Vasilakos, Multimedia services in cloud-based vehicular networks. IEEE Int. Trans. Syst. Mag. 7(3), 62-79 (2015). doi:10.1109/mits.2015.2417974 
9. Z Shen, J Luo, R Zimmermann, AV Vasilakos, Peer-to-peer media streaming: insights and new developments. Proc. IEEE. 99(12), 2089-2109 (2011). doi:10.1109/jproc.2011.2165330

10. L Zhou, H-C Chao, AV Vasilakos, Joint forensics-scheduling strategy for delay-sensitive multimedia applications over heterogeneous networks. IEEE J. Selected Areas Commun. 29(7), 1358-1367 (2011). doi:10.1109/jsac.2011.110803

11. L Zhou, N Xiong, L Shu, AV Vasilakos, S-S Yeo, Context-aware middleware for multimedia services in heterogeneous networks. IEEE Intell. Syst. 25(2), 40-47 (2010). doi:10.1109/mis.2010.48

12. Y Ye, J Sun, X Ma, AV Vasilakos, R Yuan, W Gong, Semi-random backoff: towards resource reservation for channel access in wireless LANs. IEEE-ACM Trans. Netw. 21(1), 204-217 (2013). doi:10.1109/tnet.2012. 2202323

13. Y Xiao, M Peng, J Gibson, GG Xie, D-Z Du, AV Vasilakos, Tight performance bounds of multihop fair access for MAC protocols in wireless sensor networks and underwater sensor networks. IEEE Trans. Mobile Comput. 11(10), 1538-1554 (2012). doi:10.1109/tmc.2011.190

14. PBF Duarte, ZM Fadlullah, AV Vasilakos, N Kato, On the partially overlapped channel assignment on wireless mesh network backbone: a game theoretic approach. IEEE J. Selected Areas Commun. 30(1), 119-127 (2012). doi:10.1109/jsac.2012.120111

15. J Liu, J Wan, Q Wang, P Deng, K Zhou, Y Qiao, A survey on position-based routing for vehicular ad hoc networks. Telecommun. Syst. (2015). doi:10.1007/s11235-015-9979-7

16. P Li, S Guo, S Yu, AV Vasilakos, Reliable multicast with pipelined network coding using opportunistic feeding and routing. IEEE Trans. Parallel Distributed Syst. 25(12), 3264-3273 (2014). doi:10.1109/tpds.2013. 2297105

17. Y Zeng, K Xiang, D Li, AV Vasilakos, Directional routing and scheduling for green vehicular delay tolerant networks. Wireless Netw. 19(2), 161-173 (2013). doi:10.1007/s11276-012-0457-9

18. T Spyropoulos, RN Bin Rais, T Turletti, K Obraczka, AV Vasilakos, Routing for disruption tolerant networks: taxonomy and design. Wireless Netw. 16(8), 2349-2370 (2010). doi:10.1007/s11276-010-0276-9

19. J Zhou, X Dong, Z Cao, AV Vasilakos, Secure and privacy preserving protocol for cloud-based vehicular DTNS. IEEE Trans. Inform. Forensics Secur. 10(6), 1299-1314 (2015). doi:10.1109/tifs.2015.2407326

20. A Attar, H Tang, AV Vasilakos, FR Yu, VCM Leung, A survey of security challenges in cognitive radio networks: solutions and future research directions. Proc. IEEE. 100(12), 3172-3186 (2012). doi:10.1109/jproc.2012. 2208211

21. W Viriyasitavat, M Boban, H-M Tsai, AV Vasilakos, Vehicular communications survey and challenges of channel and propagation models. IEEE Vehicular Technol. Mag. 10(2), 55-66 (2015). doi:10.1109/ mvt.2015.2410341

22. Y Toor, P Muehlethaler, A Laouiti, A de La Fortelle, Vehicle ad hoc networks: applications and related technical issues. IEEE Commun. Surv. Tutor. 10(3), 74-88 (2008). doi:10.1109/comst.2008.4625806

23. N Akhtar, SC Ergen, O Ozkasap, Vehicle mobility and communication channel models for realistic and efficient highway VANET simulation. IEEE Trans. Vehicular Technol. 64(1), 248-262 (2015). doi:10.1109/tvt.2014. 2319107

24. T Abbas, K Sjoberg, J Karedal, F Tufvesson, A measurement based shadow fading model for vehicle-to-vehicle network simulations. Int. J. Antennas Propag. (2015). doi:10.1155/2015/190607

25. P Paschalidis, K Mahler, A Kortke, M Peter, W Keusgen, in Proceedings of the 73rd IEEE Vehicular Technology Conference (VTC Spring). Pathloss and multipath power decay of the wideband car-to-car channel at $5.7 \mathrm{ghz}$, (2011), pp. 1-5. doi:10.1109/vetecs.2011.5956721

26. J Karedal, F Tufvesson, N Czink, A Paier, C Dumard, T Zemen, CF Mecklenbraeuker, AF Molisch, A geometry-based stochastic MIMO model for vehicle-to-vehicle communications. IEEE Trans. Wireless Commun. 8(7), 3646-3657 (2009). doi:10.1109/twc.2009.080753

27. V Arasan, R Koshy, Methodology for modeling highly heterogeneous traffic flow. J. Trans. Eng.-Asce. 131(7), 544-551 (2005). doi:10.1061/(asce)0733-947x(2005)131:7(544)

28. S Ukkusuri, L Du, Geometric connectivity of vehicular ad hoc networks: analytical characterization. Trans. Res. Part C: Emerging Technol. 16(5), 615-634 (2008). doi:10.1016/j.trc.2007.12.002
29. Y-C Cheng, TG Robertazzi, Critical connectivity phenomena in multihop radio models. IEEE Trans. Commun. 37(7), 770-777 (1989). doi:10.1109/26.31170

30. TK Philips, SS Panwar, AN Tantawi, Connectivity properties of a packet radio network model. IEEE Trans. Inform. Theory. 35(5), 1044-1047 (1989). doi:10.1109/18.42219

31. P Piret, On the connectivity of radio networks. IEEE Trans. Inform. Theory. 37(5), 1490-1492 (1991). doi:10.1109/18.133276

32. P Gupta, PR Kumar, in Stochastic Analysis, Control, Optimization and Applications. Systems \& Control: Foundations \& Applications, ed. by WM McEneaney, GG Yin, and Q Zhang. Stochastic analysis, control, optimization and applications, vol. Part IV, (Birkhäuser Boston, Birkhauser, Boston, 1999), pp. 547-566

33. M Sánchez, P Manzoni, ZJ Haas, in Multiaccess, Mobility and Teletraffic in Wireless Communications, ed. by E Biglieri, L Fratta, and B Jabbari. Determination of critical transmission range in ad-hoc networks, vol. 4 (Springer, Venice, Italy, 1999), pp. 293-304

34. P Santi, DM Blough, F Vainstein, in Proceedings of the 2nd ACM International Symposium on Mobile Ad Hoc Networking \& Computing. A probabilistic analysis for the range assignment problem in ad hoc networks, (Long Beach, CA, USA, 2001). doi:10.1145/501445.501446

35. B Krishnamachari, SB Wicker, R Bejar, in IEEE Global Telecommunications Conference. IEEE Global Telecommunications Conference (Globecom). Phase transition phenomena in wireless ad hoc networks, vol. 5, (San Antonio, TX, USA, 2001), pp. 2921-2925. doi:10.1109/glocom.2001.965963

36. C Bettstetter, in Proceedings of the 3rd ACM International Symposium on Mobile Ad Hoc Networking \& Computing. On the minimum node degree and connectivity of a wireless multihop network, (Lausanne, Switzerland, 2002), pp. 80-91. doi:10.1145/513800.513811

37. C Bettstetter, in Proceedings of the 56th IEEE Vehicular Technology Conference. On the connectivity of wireless multihop networks with homogeneous and inhomogeneous range assignment, vol. 3, (Vancouver Canada, 2002), pp. 1706-1710. doi:10.1109/vetecf.2002.1040507

38. C Bettstetter, J Zangl, in Proceedings of the 4th International Workshop on Mobile and Wireless Communications Network. How to achieve a connected ad hoc network with homogeneous range assignment: an analytical study with consideration of border effects, (Stockholm, Sweden, 2002), pp. 125-129. doi:10.1109/mwcn.2002.1045708

39. C Bettstetter, Topology properties of ad hoc networks with random waypoint mobility. ACM SIGMOBILE Mobile Comput. Commun. Rev. 7(3), 50-52 (2003). doi:10.1145/961268.961287

40. F Xue, PR Kumar, The number of neighbors needed for connectivity of wireless networks. Wireless Netw. 10(2), 169-181 (2004). doi:10.1023/b: wine.0000013081.09837.c0

41. M Desai, D Manjunath, On the connectivity in finite ad hoc networks. IEEE Commun. Lett. 6(10), 437-439 (2002). doi:10.1109/lcomm.2002.804241

42. HX Wang, GL Lu, WJ Jia, W Zhao, Connectivity in finite ad-hoc networks. Sci. China Series F: Inform. Sci. 51(4), 417-424 (2008). doi:10.1007/s11432008-0011-7

43. O Dousse, P Thiran, M Hasler, in Proceedings of the 21st Annual Joint Conference of the IEEE Computer and Communications Societies. Connectivity in ad-hoc and hybrid networks, vol. 2, (2002), pp. 1079-1088. doi:10.1109/infcom.2002.1019356

44. P Santi, DM Blough, in Proceedings of the International Conference on Dependable Systems and Networks. An evaluation of connectivity in mobile wireless ad hoc networks, (Washington DC, USA, 2002), pp. 89-102. doi:10.1109/dsn.2002.1028890

45. C Bettstetter, G Resta, P Santi, The node distribution of the random waypoint mobility model for wireless ad hoc networks. IEEE Trans. Mobile Comput. 2(3), 257-269 (2003). doi:10.1109/tmc.2003.1233531

46. P Panchapakesan, D Manjunath, in Proceedings of Signal Processing and Communications Conference. On the transmission range in dense ad hoc radio networks, (Bangalore, India, 2001)

47. P Santi, DM Blough, The critical transmitting range for connectivity in sparse wireless ad hoc networks. IEEE Trans. Mobile Comput. 2(1), 25-39 (2003). doi:10.1109/tmc.2003.1195149

48. CH Foh, BS Lee, in Proceedings of the 2004 IEEE International Conference on Communications. A closed form network connectivity formula one-dimensional manets, vol. 6, (2004), pp. 3739-3742. doi:10.1109/icc. 2004.1313240 
49. CH Foh, G Liu, BS Lee, B-C Seet, K-J Wong, CP Fu, Network connectivity of one-dimensional MANETs with random waypoint movement. IEEE Commun. Lett. 9(1), 31-33 (2005). doi:10.1109//comm.2005.01024

50. ZD Chen, HT Kung, D Vlah, in Proceedings of the 2nd ACM International Symposium on Mobile Ad Hoc Networking \& Computing. Ad hoc relay wireless networks over moving vehicles on highways (ACM, Long Beach, CA, USA, 2001), pp. 247-250. doi:10.1145/501449.501451

51. H Füßler, M Mauve, H Hartenstein, M Käsemann, D Vollmer, A comparison of routing strategies for vehicular ad hoc networks (2002). Technical Report TR-02-003, Department of Computer Science, University of Mannheim

52. M Rudack, M Meincke, K Jobmann, M Lott, in Proceedings of 58th IEEE Vehicular Technology Conference. On traffic dynamical aspects of inter vehicle communications (IVC), vol. 5, (2003), pp. 3368-3372. doi:10.1109/vetecf.2003.1286312

53. MM Artimy, W Robertson, WJ Phillips, in Proceedings of Canadian Conference on Electrical and Computer Engineering. Connectivity in inter-vehicle ad hoc networks, vol. 1, (Niagara Falls, Canada, 2004), pp. 293-298. doi:10.1109/ccece.2004.1345014

54. MM Artimy, WJ Phillips, W Robertson, in Proceedings of the 3rd Annual Communication Networks and Services Research Conference. Connectivity with static transmission range in vehicular ad hoc networks, (2005), pp. 237-242. doi:10.1109/cnsr.2005.29

55. MM Artimy, W Robertson, WJ Phillips, in Proceedings of IEEE Intelligent Transportation Systems Conference. Minimum transmission range in vehicular ad hoc networks over uninterrupted highways, (Toronto, Canada, 2006), pp. 1400-1405. doi:10.1109/itsc.2006.1707419

\section{Submit your manuscript to a SpringerOpen ${ }^{\circ}$ journal and benefit from:}

- Convenient online submission

- Rigorous peer review

- Immediate publication on acceptance

- Open access: articles freely available online

High visibility within the field

- Retaining the copyright to your article

Submit your next manuscript at $\gg$ springeropen.com 